To be submitted as a full paper to the Journal of Synchrotron Radiation

A BEAMLINE FOR HIGH PRESSURE STUDIES AT THE ADVANCED LIGHT SOURCE WITH A SUPERCONDUCTING BENDING MAGNET AS THE SOURCE 


\title{
A BEAMLINE FOR HIGH PRESSURE STUDIES AT THE ADVANCED LIGHT SOURCE WITH A SUPERCONDUCTING BENDING MAGNET AS THE SOURCE
}

Martin Kunz ${ }^{\mathrm{a}, \mathrm{b}}$, Alastair A. MacDowell ${ }^{\mathrm{a}}$, Wendel A. Caldwell ${ }^{\mathrm{a}, \mathrm{b}}$, Daniella Cambie ${ }^{\mathrm{a}}$, Richard S. Celestre $^{a}$, Edward E. Domninga ${ }^{a}$, Robert M. Duarte ${ }^{a}$, Arianna E. Gleason ${ }^{a, b}$, James M. Glossinger $^{a}$, Nicholas Kelez ${ }^{a}$, David W. Plate ${ }^{a}$, Tony $\mathbf{Y u}^{\mathrm{d}}$, Joeseph M. Zaug ${ }^{d}$, Howard A. Padmore $^{\mathrm{a}}$, Raymond Jeanloz ${ }^{\mathrm{b}}$, A. Paul Alivisatos ${ }^{\mathrm{c}}$, and Simon M. Clark ${ }^{\mathrm{a}}$.

\author{
.a. Lawrence Berkeley National Laboratory, 1 Cyclotron Road, Berkeley, CA 94720, USA \\ b.Department of Earth and Planetary Sciences, University of California, Berkeley, CA 94720, USA \\ c. Department of Chemistry, University of California, Berkeley, CA 94720, USA \\ d. Lawrence Livermore National Laboratory, 7000 East Avenue, Livermore, CA 94550, USA \\ *Email: mkunz@lbl.gov
}

\section{Synopsis}

A new high-pressure facility for diffraction and spectroscopy using diamond anvil highpressure cells has been developed at the Advanced Light Source. Details of the mechanics and performance of the beamline and endstation will be given.

\begin{abstract}
A new facility for high-pressure diffraction and spectroscopy using diamond anvil highpressure cells has been built at the Advanced Light Source on Beamline 12.2.2. This beamline benefits from the hard X-radiation generated by a 6 Tesla superconducting bending magnet (superbend). Useful $\mathrm{x}$-ray flux is available between $5 \mathrm{keV}$ and $35 \mathrm{keV}$. The radiation is transferred from the superbend to the experimental enclosure by the brightness preserving optics of the beamline. These optics are comprised of: a plane parabola collimating mirror (M1), followed by a Kohzu monochromator vessel with a $\operatorname{Si}(111)$ crystals $(\mathrm{E} / \Delta \mathrm{E} \sim 7000)$ and a $\mathrm{W} / \mathrm{B}_{4} \mathrm{C}$ multilayers $(\mathrm{E} / \Delta \mathrm{E} \sim 100)$, and then a toroidal focusing mirror $(\mathrm{M} 2)$ with variable focusing distance. The experimental enclosure contains an automated beam positioning system, a set of slits, ion chambers, the sample positioning goniometry and area detectors (CCD or image-plate detector). Future developments aim at the installation of a second end station dedicated for in situ laser-heating on one hand and a dedicated high-pressure singlecrystal station, applying both monochromatic as well as polychromatic techniques.
\end{abstract}




\section{Keywords: X-ray beamline, high-pressure, X-ray diffraction, superbend, diamond-anvil cell.}

\section{Introduction}

The Advanced Light Source (ALS) is a relatively low-energy $(1.9 \mathrm{GeV}), 3^{\text {rd }}$ generation synchrotron optimized for the production of VUV and soft X-ray light from undulators. However, local demand required the development of hard X-ray sources at the facility. As a result, three 6 Tesla superconducting bending magnets replaced three 1.2 Tesla warm bending magnets (Robin et al., 2002, Tamura \& Robinson, 2002). This resulted in a shift in the critical wavelength for these three sources from $3 \mathrm{keV}$ to $12 \mathrm{keV}$ allowing for the development of various hard X-ray programs. The Protein Crystallography (PX) community were the first groups to capitalize on the new hard x-ray source (Trame et al., 2003, MacDowell et al., 2004). The local high-pressure (HP) community has now followed in the installation of a dedicated beamline for high-pressure experiments. The aim of this project is to offer a state of the art user facility allowing X-ray diffraction, as well as EXAFS and X-ray imaging through a diamond anvil cell at energies up to at least $35 \mathrm{keV}$ to the high-pressure community, specifically of the North-American west-coast. It constitutes a central component within a high-pressure science centre, which ultimately will not only offer combined high-pressure / high-temperature in situ X-ray experiments, but also a range of optical techniques such as Raman-spectroscopy and Brillouin scattering. This paper describes the layout and characteristics of the high-pressure beamline and its first end-station, as well as some benchmarks relevant to X-ray diffraction experiments.

\section{Source}

The three 6 Tesla superconducting bending magnets have been operating continuously since their installation in the ring lattice in 2002. They have been transparent to the users and can be viewed as a technical success, allowing low energy rings to have access to an inexpensive hard X-ray bending magnet source and the development of the associated hard Xray programs. The design allows for 4 beamlines per magnet, with inboard and outboard pairs of tangent points at field strengths of 4.37 and 5.29 Tesla, respectively. These fields increased the critical energy from $3 \mathrm{keV}$ for a 1.27 Tesla normal conducting magnet to 10.5 and 12.7 $\mathrm{keV}$, respectively. The High Pressure beamline described in this work has a source critical 
energy of $12.7 \mathrm{keV}$. The beam size in the ALS is small, due to the small emittance of the beam $(6.75 \mathrm{~nm}-\mathrm{rad}[\mathrm{h}] ; 0.15 \mathrm{~nm}-\mathrm{rad}[\mathrm{v}])$ and the small beta functions $(0.95 \mathrm{~m}[\mathrm{~h}] ; 1.5 \mathrm{~m}[\mathrm{v}])$. Together with the small dispersion at the dipole position (dispersion $=0.57 \mathrm{~m}$; slope of dispersion $=-0.04$ ), this yields 1 sigma electron beam sizes of 98 microns [h] by 15 microns $[\mathrm{v}]$.

\section{Beamline design}

Compared to the prototype ALS superconducting bend magnet beamlines, which were optimized for the protein crystallography (PX) community (Trame at al., 2003, MacDowell et $a l ., 2004)$, the design for the HP beamline had to take into account the additional requirement of an extended energy range from the original $6-16 \mathrm{keV}$ of the PX lines to at least $35 \mathrm{keV}$. The highest flux had to be concentrated on a $100 \mu \mathrm{m}$ diameter pinhole at the sample position with a $2 \mathrm{mrad}$ horizontal convergence angle. The beamline is also required to scan in energy and be able to carry out Extended X-ray absorption Fine Structure (EXAFS) type experiments as well as X-ray-imaging techniques. Stable performance and rapid beam optimization were very important design considerations.

The beamline design is shown schematically in Fig.1. It consists of the source, a vertically deflecting plane parabolic collimating mirror (grazing angle $=2.0 \mathrm{mrad}$, acceptance $=1.0 \times 0.22 \mathrm{mrad}$ (horizontal $(\mathrm{h}) \times$ vertical $(\mathrm{v})$ ) that provides parallel radiation in the vertical for a double crystal monochromator (two flat crystals or two flat multilayers), followed by a toroidal focusing mirror, the sample and backstop. For this beamline (ALS nomenclature 12.2.2) the various distances of the beamline components from the source are 6.5, 16.5, 18.8 and $28.2 \mathrm{~m}$ for the plane parabola, monochromator, toroid and sample, respectively (Fig. 1). This optical arrangement uses the toroid in the 2:1 horizontal demagnification with the result that astigmatic coma is eliminated (MacDowell et al., 2004) and a focus spot of high fidelity is achieved. Raytracing using Shadow (Lai et al., 1986) indicated a focus spot size of 153 x 64 $\mu \mathrm{m}$ full width half maximum (fwhm) with a beamline acceptance angle of $1.0 \times 0.22 \mathrm{mrad}$ (horizontal x vertical) and a source size of $220 \times 30 \mu \mathrm{m}$ fwhm. The raytrace is with perfect optics of zero slope error. Real optics are imperfect with slope errors which will degrade the spot size. To achieve a spot size of $150 \times 100 \mu \mathrm{m}$ fwhm (h x v) requires optical slope error tolerances of $<1 \mu \mathrm{rad}$ (MacDowell et al., 2004). Such tolerances require very high quality optics near the limit of the current manufacturing capability. 


\subsection{Optics}

The description of the individual optics is as follows. The M1 plane parabola is a flat side cooled silicon mirror assembled into a mechanical bender. Cooling was achieved using water cooled copper bars held along the mirror sides with a 50-100 $\mu \mathrm{m}$ gap filled with gallium-indium-tin eutectic liquid metal. The parabolic shape of the M1 mirror was set using the ALS Long Trace Profiler (Irick, 1994). Metrology measurements indicated a slope error of $0.7 \mu \mathrm{rad} \mathrm{rms}$ and a finish of $0.25 \mathrm{~nm} \mathrm{rms}$.

Following the pre-mirror is a standard APM Kohzu monochromator with custom inhouse additions for the crystals and multilayer optics. Fig. 2 shows the schematic layout. The basic monochromator rotates all optics about the central axis that runs along the surface of the first crystal. This rotation is the main Theta drive that determines the monochromator angle and thus X-ray energy selected. The first crystal is fixed to this main rotation platform, whilst the second crystal is mounted off a stack of 4 stages that in turn is supported from the main Theta rotation platform. The 4 stages that the second crystal rides on can be adjusted to maintain the constant beam height offset required for the beamline optics. The first multilayer is fixed to the first crystal support structure and as shown in Fig.2 is mounted upstream of the first crystal. The second multilayer is mounted on the stack of 4 stages along with the second Si(111) crystal. By rotating the main Theta drive to the low grazing angle required for the multilayer, the off axis location allows it to intercept the beam before the $\mathrm{Si}(111)$ crystal and directs the x-rays to the second multilayer (see Fig. 2). Of course the off axis location of the first multilayer means that the x-rays will walk off the surface, but by suitable choice of mirror length $(176 \mathrm{~mm})$ and $\mathrm{d}$ spacing $(2.0 \mathrm{~nm})$ it is possible to get a useful energy range of 14- $29 \mathrm{keV}$ before the beam has walked too far off the mirrors. The multilayers used were 150 layer pairs of $\mathrm{W} / \mathrm{B}_{4} \mathrm{C}$ supplied by Osmic Inc. with figure error $<1 \mu \mathrm{rad} \mathrm{rms}$.

The maximum absorbed power density on the multilayer mirrors at $18 \mathrm{keV}$ is calculated to be 1.65 watts $/ \mathrm{cm}^{2}$. Finite element analysis indicated that side cooling of the first multilayer optic would be adequate to retain the figure error $<1$ urad rms. Side cooling was achieved by clamping water-cooled copper plates to both sides of the mirror with a gallium/indium/tin eutectic thermal interface.

For the flat $\mathrm{Si}(111)$ crystal the power load is 46 watts with a maximum power density of 15.2 watts $/ \mathrm{cm}^{2}$ at $12.4 \mathrm{keV}$. At these power levels, internal water-cooling is adequate, but care has to be taken with the design of the water channels. A design similar to that used before was employed (MacDowell et al. 2004) but the increased high energy of this beamline compared to the PX beamline precluded the use of epoxy for attaching the invar water 
plenums to the silicon crystal as calculations (Howells, 1999) indicated that the epoxy lifetime would be $<1$ year. A low temperature solder procedure was developed to attach the water plenums to the crystals. The thermal expansion of the silicon and invar are slightly different (2.0 $\mathrm{ppm} / \mathrm{C}$ and $0.5 \mathrm{ppm} / \mathrm{C}$ respectively), so we minimize the strain introduced by the temperature excursion during the soldering process by simply lowering the soldering liquidus temperature. The procedure was as follows. The sides of the silicon crystal and mating surface of the invar water plenum were lapped flat and then coated with $100 \mathrm{~nm}$ of gold by evaporation with a $5 \mathrm{~nm}$ chromium under layer. The chosen solder consisted of $57 \%$ bismuth, $26 \%$ indium and 17\% tin (Type number 122801) from Indium Corporation of America selected for its low melting temperature of $78^{\circ} \mathrm{C}$. The pieces were assembled at $90-100^{\circ} \mathrm{C}$ on a hot plate and worked with an ultrasonic soldering iron to help break up oxide layers and ensure a reliable mechanical solder seal. Subsequent rocking curve measurements during beamline commissioning did not indicate crystal strain was a problem for the $\mathrm{Si}(111)$ rocking curve widths.

The second silicon crystal and multilayer optics have the shape of a rectangular parallelepiped ('brick'). The last optic is a silicon cylinder supplied by Seso Inc. and bent into a toroid by a mechanical bender. Slope errors on this unbent optic were measured as $1.4 \mu \mathrm{rad}$ $\mathrm{rms}$ and a roughness of $<0.4 \mathrm{~nm}$ rms. Both the M1 and M2 mirrors were bent with cantilever style spring bending mechanisms (Howells et al., 2000).

The coating of the M1 mirror was $8 \mathrm{~nm}$ rhodium over $25 \mathrm{~nm}$ platinum over a $5 \mathrm{~nm}$ chromium "glue" layer. A similar coating was used for the M2 mirror except the rhodium coating was $4 \mathrm{~nm}$ thick rather than $8 \mathrm{~nm}$ thick. The reflectivity of the 2 different coatings along with the reflectivity of rhodium and platinum for a grazing angle of $2 \mathrm{mrad}$ is shown in Fig.3. The single platinum coating produces good reflectivity to high energy but has contaminating L edges in the 11-14 keV range. The use of a rhodium over platinum bilayer mirror allows for suppression of the platinum L edges as these low energy x-rays are reflected from the rhodium layer. However the high energy x-rays penetrate the rhodium and are reflected from the underlying platinum layer. Thus this bilayer has a higher cut off energy when compared to just a rhodium mirror at the same grazing angle. Fig. 2 suggests the energy range is extended $\sim 5 \mathrm{keV}$ compared to a single rhodium layer. The reason for the difference in coating of the 2 mirrors is historical as the M1 mirrors were supplied as a batch for the superbend protein crystallography project (MacDowell et al., 2004). For this project the 8nm rhodium thickness was more appropriate for the higher grazing angle of $4.5 \mathrm{mrad}$. For the more grazing angle of 
$2 \mathrm{mrad}$ required for this High Pressure beamline, the thinner 4nm rhodium layer was more appropriate, but not to the extent of recoating the M1 mirror.

\subsection{Beam Position Feedback Scheme}

The focused spot size at the sample is comparable with the sample size, which is typically less than the $150 \mu \mathrm{m}$ inside diameter of the metal-gasket in the diamond anvil cell. Typically beamlines suffer from slow thermal variations of the beamline components, beam drifts and other unspecified environmental drifts that cause fluctuations in intensity and beam position at the sample. The requirement is for the beam to remain stable on the same position on the sample for at least the time it takes to record the required data. To solve the drift problems, we initially adopted a feedback system based on the horizontal and vertical positional information from a fluorescence detector equipped with 4 PIN diodes that give beam positional information (Alkire, et al. 2000). The detector is located $70 \mathrm{~cm}$ before the focus position. This detector proved to have a non linear response with photon energy leading it to give an erroneous signal on which to provide beam position feedback. The source of this non linear response is still under investigation but initial work suggests that the signal level (typically $<0.1 \mathrm{nA} @ 12 \mathrm{KeV}$ ) is rather small compared to the noise and electronic drift levels and this poor signal to noise ratio is an inherent problem for this device in this beamline.

An alternative feedback scheme was used (MacDowell et al., 2004). A cerium-doped yttrium aluminum garnet (YAG) scintillator is glued to the shutter blade of the CCD shutter (nm-Laser) located $50 \mathrm{~cm}$ before the sample. The scintillated beam image of the beam is viewed with a TV camera. This image is fed to a frame grabber that determines the beam position parameters at $5 \mathrm{~Hz}$. Vertical beam position is affected by movement of the M2 tilt, and horizontal beam position changes by rolling the second monochromator crystal. Beam position centroid stabilities of $+-5 \mu \mathrm{m}$ are typically achieved. The drawback of this control system is that it is only operational when the X-ray shutter is closed. When the shutter is open, the system operates in open-loop mode. However, the beamline has proved to be stable to the level of $\sim 20 \mu \mathrm{m}$ over the typical longer recording times of $\sim 5 \mathrm{~min}$. The beamline has proved to be more stable in this open loop mode compared to the PX beamlines. It is surmised that the reduced power loading on the M1 mirror for the HP beamline due to the $2.0 \mathrm{mrad}$ grazing angle helps with overall beamline stability compared to the PX beamlines which operate at $4.5 \mathrm{mrad}$ grazing angle. In order also to provide beam stabilization during longer ( $>5 \mathrm{mins}$ ) 
exposure cycles, we adopted a combined scheme using the YAG scintillator as well as the fluorescence foil. The YAG scintillator is the dominant stabilizer. Whenever the shutter is closed, i.e. the YAG scintillator is brought into the beam, the beam is returned to its stored optimal position. When opening the shutter, the system records the current fluorescence values and keeps these constant as long as the shutter remains open. This procedure works fine for monochromatic diffraction experiments where the wavelength is fixed.

\subsection{End Station}

In order to cover the expected range of experimental flexibility, two end-stations are installed on a $3.6 \mathrm{~m} \mathrm{x} 1.2 \mathrm{~m}$ optical table, which in turn is placed within a spacious $(5.4 \mathrm{~m} \mathrm{x}$ $3.2 \mathrm{~m}$ ) hutch. After entering the hutch, the beam is conditioned and controlled by a set of absorption foils, a fast shutter, a set of horizontal and vertical aperture slits and a clean-up pinhole. The absorption foils $(\mathrm{Cu}, \mathrm{Zn}, \mathrm{Pd}, \mathrm{Sn}, \mathrm{Ag}$ and $\mathrm{Mo})$, purchased from EXAFSMaterials, can be used for intensity attenuation at various energies and to calibrate the monochromator. This is routinely done at the beginning of a new experiment, although tests during commissioning have shown the energy to be stable within $2 \mathrm{eV}$ over the period of weeks of frequent energy changes. The fast shutter is water cooled and interfaced with the area detectors. A YAG scintillating crystal mounted on the front of the fast shutter allows for beam-position stabilization (see above). The slits and clean-up pinhole allow shaping the beam to the desired size. A clean, circular beamspot is important for high-pressure experiments where even the smallest amount of X-rays falling on the highly scattering steel or rhenium gasket material can create intense background signals. Centering a $100 \mu \mathrm{m}$ gasket hole onto a $100 \mu \mathrm{m}$ beam requires easy and fast pre-centering procedure. We solved this by a mirror prism assembly (Fig. 4) mounted on a drive holding several pre-aligned tantalum pinholes. A parallax-free image of the diamond anvil cell is viewed via a video camera A small hole in the center of the mirror-prism ensures un-attenuated X-ray transmission without reducing the quality of the optical image. The DAC is moved to the pre-aligned beam position center and then the mirror prism is moved out of the beam and replaced by a pre-aligned Tapinhole acting as a scatter guard (Fig. 4).

Of the two planned end-stations, the first one has been commissioned and tested. Its present design is laid out for high-pressure powder diffraction in a diamond anvil cell (DAC), mostly aiming at measuring accurate cell parameters to determine equations of state of solid (and liquid) material. Samples are mounted in a diamond anvil cell onto a simple goniometer. The goniometer consists from bottom to top of the following stages (Fig. 5): 1) a horizontal 
translation stage perpendicular to the X-ray beam (x-direction); 2) a rotation stage with its axis vertical; 3) a vertical stage (y-direction), 4) a second horizontal-translation stage perpendicular to the beam (x-direction) and 5) a horizontal translation stage parallel to the beam (z-direction). This arrangement enables a) centering the vertical rotation axis onto the beam using stage 1) and b) placing the sample onto the rotation axis and beam using stages 4 ) and 5). This scheme allows to use the rotation axis as a reference position relative to the detector plane, and thus to precisely determine the sample to detector distance (STD). Knowing this distance accurately is crucial for powder diffraction experiments, since the relative error in $\mathrm{d}$-spacings (and thus cell parameters) $\Delta \mathrm{d} / \mathrm{d}$ scales linearly with the relative error in distance. Thus to achieve a desired accuracy of $\Delta \mathrm{d} / \mathrm{d}$ of $10^{-4}$ at a distance of $100 \mathrm{~mm}$, the sample to detector distance needs to be known to better than $10 \mu \mathrm{m}$. To determine the $\mathrm{STD}$, we first place a calibrant (e.g. $\mathrm{LaB}_{6}, \mathrm{Si}, \mathrm{NaCl}$ ) onto the rotation axis and take a diffraction pattern. The known lattice parameter of the calibrant, together with the independently determined wavelength (by absorption edge, see above) allows us to determine the distance between the detector plane and the rotation axis from the diffraction pattern of the calibration material. This distance will then correspond to the STD if the DAC with sample is placed on the rotation axis as well. In order to center the calibrant and DAC onto the rotation axis, the absorption profile of the gasket hole containing the sample is scanned along the sample $\mathrm{X}$-stage (4 in Fig. 5) across the X-ray beam at two different rotation-angles. The shift of the gasket hole along the $\mathrm{x}$-stage $(\mathrm{dx})$ due to the rotation divided by the rotation angle ( $\tan$ $\omega)$ gives the off-set of the sample from the rotation axis parallel to the beam ( $\mathrm{z})$ as $z=d x / \tan$ $\omega$ (Fig. 6). With this approach, samples can be placed onto the rotation axis of end station 1 with a reproducibility of better than $8 \mu \mathrm{m}$.

A Mar345 image plate detector (circular active area of $345 \mathrm{~mm}$ diameter, readout-time $\sim 40$ secs) or a Bruker Smart CCD (square active area of $100 \mathrm{~mm}$ edge length, read out time $1 \mathrm{sec}$ ) are used for detectors. Users tend to prefer the larger sized, slower image plate reader over the smaller and faster CCD detector despite the reduced flexibility in sample to detector distance. The instrumental resolution of the end-station depends on the divergence of the incident X-rays, size of the beam, thickness of the sample, diffraction angle, sample to detector distance (STD) and points-spread function of the detector. Assuming a point-spread function of $\sim 0.25 \mathrm{~mm}$ for the CCD detector and a divergence of $0.5 \mathrm{mrad}$, we calculate a theoretical FWHM of $0.15^{\circ}$ at $100 \mathrm{~mm}$ STD, which compares well with the observed value of $0.16^{\circ}$. 


\section{Beamline Performance}

The size of the collimated beam was measured by scanning a single blade of the slit assembly across the optimized beam. Beamline acceptance was 1.0 x $0.22 \mathrm{mrad}(\mathrm{h} \mathrm{x} \mathrm{v})$. The measured size of $115 \times 78 \mu \mathrm{m}$ fwhm compares with the raytraced theoretical value of $153 \times 64 \mu \mathrm{m}$. The slightly larger spot size in the vertical can be attributed to the various summation of figure errors of the optics. The smaller size in the horizontal is less easy to explain. The current optical scheme is designed to eliminate astigmatic coma, (MacDowell et al., 2004) and so minimize the vertical beam size. Small changes in the grazing angles of M1 and M2 break this condition allowing for other aberrations to balance off against each other. Preliminary raytracing work has shown there are slight improvements in the horizontal focused size by doing this.

The calculated and measured fluxes arriving at the sample with and without a pinhole collimator are shown in Fig. 7. The electron source used has an energy of $1.9 \mathrm{GeV}$ within a field of 5.27 Tesla at the tangent point. The absolute flux was measured by using an ion chamber (IC). The IC has a total length of $17 \mathrm{~cm}$, an active length of $14.7 \mathrm{~cm}$ (guard electrodes occupied the $1.15 \mathrm{~cm}$ end sections) and is filled with 1 bar of $\mathrm{Ar}$ gas. For measurements of the multilayer-flux, a $3.2 \mathrm{~mm} \mathrm{Al}$ foils were inserted into the beam in order to avoid saturation of the IC. The current measured in the ion chamber is converted into number of $\mathrm{x}$-ray photons, using a conversion factor of $26 \mathrm{eV}$ of energy per electron - ion pair (Thompson, 2001). The photon flux derived from the ion chamber is checked against a silicon photodiode detector signal, which in turn was calibrated against a (NaI(Tl)) scintillator/photomultiplier-tube pulse counting detector (Bicron model 1XMP.040B) operating at $<10^{5} \mathrm{~Hz}$. The low rate of the scintillator detector allows for the assumption of zero dead time and thus linear response. The scintillator detection efficiency is considered to be 1.0 (Holl et al., 1988). The result of the flux measurements is compared against the expected values in Fig. 7. The calculated total flux from the $\mathrm{Si}(111)$ crystals (no pinhole) is about twice the value measured. The reason for this discrepancy is still under investigation. With the insertion of a $100 \mu \mathrm{m}$ pinhole the flux drops to $44 \%$ of the no pinhole condition. Raytracing indicates that the flux reduction should be to $36 \%$. The smaller discrepancy between the flux measured and calculated through the pinhole is associated with the smaller spot size observed compared to the raytraced version (see above). At high energies, the flux is increasingly difficult to measure due to the decreasing efficiency of both the ion-chamber and silicon-diode detectors. The flux plots thus terminate a $35 \mathrm{keV}$, and no roll off due to exceeding the critical angles of the mirrors is observed. The critical angle of the beamline is 
therefore slightly higher consistent with reflectivity plots of Fig. 3. For the multilayer, we observe calculated and measured flux to be similar ( $<$ factor 1.5) over the multilayer range. The rapid drop off of flux at either end of its range is due to the beam walking off the multilayer mirrors with energy change. Energy resolution of the 150 layer multilayer monochromator was measured at the rhodium edge as $\mathrm{E} / \Delta \mathrm{E}=120$.

Two benchmarks for experiments on ES-1 were determined: 1) The correlation between pressure determined using spectroscopic methods as compared to an internal diffraction standard. This gives us an indication on the reliability of compressibility measurements on 12.2.2. 2) The integral uncertainty on refinable parameters of a Rietveld refinement (atomic coordinates). This gives us a benchmark on the best possible accuracy for structural measurements.

Benchmark 1): Pressure in a diamond anvil cell can be determined by measuring the pressure-induced shift of the laser-excited fluorescence shift of a ruby grain within the pressure camber (spectroscopic method) (e.g. Piermarini et al., 1975). Alternatively, the known equation of state (EoS) of a calibrant allows using its diffraction signal to deduce the pressure within a diamond anvil cell (internal standard). Comparing the pressures derived from $\mathrm{NaCl}$-volumes against the ruby pressure gauge therefore gives us an indication on the reliability of compressibility data measured on end-station 1 . To do this, a series of different ruby calibrations applied to our spectroscopic measurements (Piermarini et al., 1975; Mao et al., 1986; Holzapfel, 2003, Dorogokupets and Oganov, 2003) were compared against NaClvolumes measured on end station 1 and converted into pressure using three different $\mathrm{NaCl}$ equations of state (Decker, 1971; Birch, 1986; Brown, 1999). $\mathrm{NaCl}$ powder together with 8 different ruby grains were loaded into a Diacell membrane driven DAC. Ethyl Cyclo Hexane $(\mathrm{ECH})$ was used as pressure medium. ECH was preferred to the usual Ethanol-Methanol mixture since many users are working with hygroscopic samples. The hydrostaticity of ECH is comparable to that of ethanol - methanol. Standard deviations for the spectroscopic data were determined from 5 to 8 different measurements on different ruby grains. Volumes were calculated by fitting each of the first seven $\mathrm{NaCl}$ peaks individually using a Voigt function as implemented in fit2d (Hammersley, 1997) and converting each measurement into a volume. The seven volumes were used to calculate an average value and an estimated standard deviation. All measurements were performed at room-temperature. The general agreement between the ruby scales and the $\mathrm{NaCl}$ pressure is good, which gives confidence in the capability of the beamline to produce reliable compressibility data. This is supported by a 
closer look at the data (Figure 8): Very good correspondence (mean deviation 0.2 GPa) is observed for the EoS given by Decker (1971), and the ruby scale proposed by Piermarini et al. (1975) (Fig. 8). Since the Piermarini scale is gauged solely against the Decker equation of state, these two scales should show identical pressures, provided the volumes extracted are reliable. The good agreement between these two scales found on our beamline, confirms therefore the internal consistency of the $\mathrm{NaCl}$ compressibility measured on 12.2.2 end station 1. Good agreement is also observed between Birch's EoS and the ruby scale proposed by Mao et al.(1986), Holzapfel (2002) and Dorogokupets and Oganov (2002). All of these ruby scales were calibrated against the compressibility of a series of different metals. The Brown (1999) compressibility model seems to be in best agreement with Piermarini's ruby scale.

Benchmark 2). It is well known that estimated standard deviations (esd's) of atomic coordinates and cell parameters, derived analytically from Rietveld refinements tend to be underestimated (e.g. Hill, 1991). In order to establish an integral set of standard deviation for 12.2.2 ES-1, a powder diffraction experiment on a standard $\left(\mathrm{LaB}_{6}\right)$ in air was repeated 9 times including the subsequent data reduction and analysis with fit2d (Hammersley, 1997; Hammersley et al., 1996)) and GSAS (Larson and Von Dreele, 2000; Toby, 2001). LaB 6 was used since it is known to have very low strain-broadening and is therefore used as a standard in powder diffraction (NIST standard 660 a). The experiments were conducted at an energy of $25.514 \mathrm{keV}(\lambda=0.48593 \AA)$. This specific energy was chosen since it corresponds to the $\mathrm{Ag}$ K-edge and therefore allowed for a precise wavelength calibration independent of the diffraction experiment. A Smart 6000 CCD (active area $10 \times 10 \mathrm{~cm}$, pixel size $\sim 0.1 \times 0.1$ $\mathrm{mm}$ ) was used as detector. Frames were corrected for dark current as well as spatial distortion using the Smart software. For each exposure, the sample (Sigma Aldrich 24,185-7, Lot-\# MU 04702MR), loaded into a gasket hole of $0.15 \mathrm{~mm}$ diameter and mounted outside a DAC onto the goniometer. After each exposure, the sample was taken off the beam line, re-mounted on its holder and placed back on the goniometer before applying the centering routines described above. Each of the resulting datasets was first used to determine a sample to detector distance (STD) using the cell-parameters given by the National Bureau of Standards (4.1569 $\AA$ ) (NIST SRM 660-a) The STD, which was used to convert the 2-dimensional diffraction images into 1-dimensional 2-theta vs. intensity plots, was taken from the average of these 9 individual measurements $(99.949 \mathrm{~mm})$. For the Rietveld refinement in GSAS, lattice parameter, zeropoint, background parameters (Shifted Chebyeshev with 12 parameters), peak profile parameters (6 parameters of a pseudo voigt function) as well as the X-coordinate of the Batom and an isotropic displacement parameter $\left(\mathrm{U}_{\text {iso }}\right)$ for La and $\mathrm{B}$ were refined. The results are 
summarized in Table 1. As can be seen, our diffraction system produces lattice parameters with an internal precision of $4 \cdot 10^{-4}$. This figure could be improved by a factor of 2 or 3 by using a larger detector at a longer STD. When comparing our refined cell parameter to the theoretical value published by NIST SRM-660a, we find a striking difference of 4.167(2) $\AA$ vs $4.157 \AA$, i.e. about 5 times the estimated standard deviation. We attribute this discrepancy to a real discrepancy between our sample and the NIST sample. Such a discrepancy cannot be fully compensated in the distance calibration procedure since the effect of a different lattice parameter varies with $\sin (\theta)$, whereas the STD affects peak position as a function of $\tan (\theta)$. A full profile refinement including a zero-point will therefore reflect this discrepancy. Forcing the zero-point to a value of zero was not an option, because it prevented the Rietveld refinement from convergence due to large positional mismatches at high angles. Inter-atomic distances are affected by both peak position and peak intensity. In our case, the La-B distance is determined by the lattice parameter on one hand and a single free coordinate $\mathrm{x}(\mathrm{B})$ on the other hand. Here we find a precision of $2 \cdot 10^{-3}$. Despite the fact that we observe a larger cell parameter than the NIST value, our La-B distance $[3.050(7) \AA]$ is within one esd to the NIST value (3.054 $\AA$ ). Although it is very difficult to extract meaningful displacement parameters from Rietveld refinements, refined $U_{\text {iso' }} s$ can still give an indication on the quality of the intensity data. As shown in Table 1, all $\mathrm{U}_{\text {iso }}$ refined to positive values, which are in a physically meaningful order of magnitude. This lends confidence in the measured intensities, although we do not aim at interpreting displacement parameters from high-pressure powder diffraction experiments.

\section{Control System}

The success of beamlines is becoming more dependent on their ease of use, which is strongly influenced by the control software. This high-pressure facility employs separate control systems for the beamline and end station. The beamline is operated by a now standard ALS control system based on Labview (National Instruments). All hardware and software components are modular, because most beamlines contain a limited set of relatively standard components that are independent of the experimental end station. This beamline control system can operate stand-alone and be used to commission the beamline without a functioning end station. Following commissioning, the beamline control system can be put into a driven mode and receive control inputs via the local network from the end station control system. 
Such commands can shift the energy, control the beam convergence onto the sample and tune up the beamline.

The end station control code is also an in-house control system based on Labview. However the ever changing requirements of the experiments require continued modifications to the end station control system. This takes significant effort if the procedure is to modify the end station code each time a change is required. To address this issue the software is to have the higher level macro system developed on top of the current system that will allow users to modify the control as required for the separate experiments. This development is underway.

\section{Future Developments}

The hutch was designed to be long enough so as to accommodate a second end station. Slits at the location of the first station will act as a virtual source that will be re-imaged by KB mirrors with a demagnification 2 × 6 (h x v) onto a second station. By adjusting the slits at the virtual (secondary) source a spot size of $\sim 10 \mu \mathrm{m}$ on the sample is expected. This upgrade is underway. The second station is to be equipped with a double-sided laser heating system.

Once the laser heating set-up is installed and commissioned, we plan to replace the powder diffraction set-up of end-station 1 with a single-crystal diffractometer. The photon flux and energy spectrum provided by a super-conducting bending magnet provides an excellent source to interface diamond anvil cells with single-crystal X-ray diffraction. This is not only of high interest for the local high-pressure community but is also in line with recent international developments in high-pressure crystallography. The quest for higher and higher pressures involves smaller and smaller sample volumes, which in turns results in poorer powder statistics, thus imposing an intrinsic pressure limit for powder crystallography: It can be shown that, in order to maintain a measured accuracy on powder diffraction intensities of $5 \%$, the number of grains required is about $10^{6}$. This in turn poses a limit on minimal sample volume and thus maximal pressure if one assumes a minimal acceptable grain size $(\sim 1$ micron). In order to overcome this limitation, we plan to revitalize polychromatic singlecrystal diffraction. We intend to develop a scanning monochromator technique in order to circumvent familiar problems of Laue diffraction (harmonic overlap, energy-specific absorption correction). Alternatively we also envisage exploiting the wide bandpass of the multilayer monochromator. This offers a pink-beam, which maybe also used to solve the above-mentioned problem of harmonic overlap. In any case, an approach using polychromatic 
single crystal diffraction will facilitate the extraction of structural data from static samples and is thus ideal to be combined with the presently installed laser heating set-up.

\section{Conclusions}

End station 1 of beamline 12.2.2 of the Advanced Light Source offers an experimental facility dedicated to high-pressure research. Its energy range and flux permit X-ray powder diffraction and X-ray spectroscopic experiments between $5-35 \mathrm{keV}$ up to pressures of $>50 \mathrm{GPa}$ and temperatures of $800 \mathrm{~K}$. This demonstrates that with state of the art beamline technology, competitive high-pressure research can be conducted at a $1.9 \mathrm{GeV}$ storage ring. Flux and energy range of this type of beamline are ideal for future developments in the rising field of synchrotron based high-pressure single-crystal X-ray diffraction.

\section{Acknowledgements}

We would like to thank the many staff at the Lawrence Berkeley National Laboratory who have contributed to this project. We would also like to thank the many beamline users who have borne with us through all of the teething problems and who's suggestions have contributed greatly to the final configuration of this beamline. We would like to thank Tomas Diaz De La Rubia and Lou Terminello at the Lawrence Livermore National Laboratory for contributions of equipment that enabled us to complete and enhance end station 1.

The Advanced Light Source is supported by the Director, Office of Science, Office of Basic Energy Sciences, Materials Sciences Division, of the U.S. Department of Energy under Contract No. DE-AC03-76SF00098 at Lawrence Berkeley National Laboratory and University of California, Berkeley, California. COMPRES, the Consortium for Materials Properties Research in Earth Sciences under NSF Cooperative Agreement EAR 01-35554 supported this project through funding of MK, WAC and AEG as well as crucial beamlineequipment. 


\section{References}

Alkire, R.W., Rosembaum, G., and Evans, G., (2000) J.Sync. Rad. 7, 61-68

Birch, F. (1986), Journal of Geophysical Reserch, 91 (B5), 4949 - 4954.

Brown, M.J. (1999), Journal of Applied Physics, 86 (10), 5801 - 5808.

Decker, D.L. (1971), Journal of Applied Physics, 42 (8), 3239 - 3244.

Dorogokupets, P.I. \& Oganov, A.R. (2003). Doklady Earth Sciences, 391A (6), 854857.

Hammersley, A.P., Svensson, S.O., Hanfland, M., Fitch, A.N., and Häusermann, D. (1996): Two-Dimensional Detector Software: From Real Detector to Idealised Image or Two-Theta Scan, High Pressure Research, 14, 235-248.

Hammersley, A.P. (1997): ESRF Internal Report, ESRF97HA02T, “FIT2D: An Introduction and Overview", (1997)

Hill, R.J. (1991) 'Data collection strategies: fitting the experiment to the need.' In: The Rietveld Method. Young, R.A. editor, Oxford University Press, Oxford.

Holl, I., Lorenz, E. and Mageras, G. (1988) IEEE Tans. Nuclear Science, 35 105-109. This paper indicates that the visible photon yield from $\mathrm{NaI}(\mathrm{Tl})$ scintillator is 38 photons $/ \mathrm{KeV}$ when irradiated with $\mathrm{x}$-rays. We assume a conservative light coupling of these photons into the photomultiplier tube of $\sim 50 \%$. The efficiency of the Hamamatsu R580 photomultiplier tube is $\sim 25 \%$ (http://usa.hamamatsu.com) at the emission wavelength of $\mathrm{NaI}(\mathrm{Tl})-430 \mathrm{~nm}$. A $12 \mathrm{KeV}$ $\mathrm{x}$-rays is expected to generate $\sim 57$ electrons/ $\mathrm{x}$-ray from the photocathode before entering the electron multiplier section. The scintillator photomultiplier combination is thus expected to single count $\mathrm{x}$-rays with a quantum efficiency of 1.0 after the absorption by the $125 \mu \mathrm{m}$ thick beryllium entrance window is taken into account.

Holzapfel, W.B. (2003). Journal of Applied Physics. 93(3), 1813-1818.

Howells, M.R. (1999). Advanced Light Source Notes. LSBL-529

Howells, M.R., Cambie, D., Duarte, R., Irick, S., MacDowell, A.A., Padmore, H.A., Renner, T., Seungyu, R. \& Sandler, R. (2000). Optical Engineering. 39, 2748-2762.

Irick, S., (1994), Nucl. Inst. Meth., A347, 226-230

Lai, B. \& Cerrina, F. (1986). Nucl. Instrum. Meth. A246, 337-341.

Larson, A.C. and Von Dreele, R.B. (2000): General Structure Analysis System (GSAS), Los Alamos National Laboratory Report LAUR 86-748 (2000).

MacDowell,A.A., Celestre' R.S., Howells M.R., McKinney W., Krupnick J., Cambie D., Domning E.E, Duarte R.M., Kelez N., Plate D.W., Cork C.W., Earnest T.N., Dickert J., Meigs, G., Ralston C., Holton J.M., Alber T., Berger J.M., Agard D.A., \& Padmore H.A., (2004). J. Sync. Rad., 11, 447-455. 
Mao, H.-K., Xu, J. \& Bell, P.M. (1986). Journal of Geophysical research - Solid Earth and Planets. 91 B5, $4673-4676$.

Piermarini, G.J., Block, S., Barnett, J.D. \& Forman, R.A. (1995). Journal of Applied Physics. 46(6), 2774-2780.

Robin, D., Benjegerdes, R., Biocca, A., Bish, P., Brown, W., Calais, D., Chin, M., Corradi, C., Coulomb, D., De Vries, J., DeMarco, R., Fahmie, M., Geyer, A., Harkins, J., Henderson, T., Hinkson, J., Hoyer, E., Hull, D., Jacobson, S., Krupnick, J., Marks, S., McDonald, J., Mollinari, P., Mueller, R., Nadolski, L., Nishimura, K., Ottens, F., Paterson, J.A., Pipersky, P., Ritchie, A., Rossi, S., Salvant, B., Schlueter, R., Schwartz, A., Spring, J., Steier, C., Taylor, C., Thur, W., Timossi, C., Wandesforde, J. \& Zbasnik, J..(2002). J. Proceedings of the 2002 European Particle Accelerator Conf., Paris, France, 215-217.

Tamura, L. \& Robinson, A. (2002). Synchrotron Radiation News, 15, 30-34.

Thompson, A.C. (2001). X-ray Data Booklet, $2^{\text {nd }}$ edition, edited by A.C. Thompson and D. Vaughan, p 4-35, LBNL/PUB-490 Rev.2, Berkeley.

Toby, B.H. EXPGUI, a graphical user interface for GSAS, J. Appl. Cryst. 34, 210-213 (2001).

Trame, C, MacDowell, A.A., Celestre, R.Padmore, H.A.Cambie, D., Domning, E.E., Duarte, R.M., Kelez, N., Plate, D.W., Holton, J.M., Frankel, K., Tsutakawa S., Tainer J., \& Cooper, P.K. (2004). AIP Conf Proc. 705, 502-505. 


\section{DISCLAIMER}

This document was prepared as an account of work sponsored by the United States Government. While this document is believed to contain correct information, neither the United States Government nor any agency thereof, nor The Regents of the University of California, nor any of their employees, makes any warranty, express or implied, or assumes any legal responsibility for the accuracy, completeness, or usefulness of any information, apparatus, product, or process disclosed, or represents that its use would not infringe privately owned rights. Reference herein to any specific commercial product, process, or service by its trade name, trademark, manufacturer, or otherwise, does not necessarily constitute or imply its endorsement, recommendation, or favoring by the United States Government or any agency thereof, or The Regents of the University of California. The views and opinions of authors expressed herein do not necessarily state or reflect those of the United States Government or any agency thereof or The Regents of the University of California. 
Table 1: Refined values for cell parameter $(a)$, isotropic displacement parameters $\left(U_{i s o}\right)$ and interatomic distance $(\mathrm{La}-\mathrm{B})$ from 9 independent powder diffraction experiments on $\mathrm{LaB}_{6}$ (NIST SMT 660a).

\begin{tabular}{ccccc}
\hline Number & $\mathbf{a}(\mathbf{A})$ & $\mathbf{U}_{\text {iso }}(\mathbf{B})(\hat{\mathbf{A}})^{2}$ & $\mathbf{U}_{\text {iso }}(\mathbf{B})(\mathbf{A})^{2}$ & La-B (A) \\
\hline 1 & 4.167960 & 0.00418 & 0.00424 & 3.0495 \\
2 & 4.163424 & 0.00563 & 0.00330 & 3.0454 \\
3 & 4.167552 & 0.00598 & 0.00386 & 3.0448 \\
4 & 4.168971 & 0.00209 & 0.00834 & 3.0420 \\
5 & 4.168985 & 0.00138 & 0.00341 & 3.0474 \\
6 & 4.168031 & 0.00302 & 0.00857 & 3.0479 \\
7 & 4.167924 & 0.00279 & 0.00269 & 3.0532 \\
8 & 4.165755 & 0.00274 & 0.00678 & 3.0500 \\
9 & 4.167202 & 0.00214 & 0.00365 & 3.0680 \\
\hline Average & $4.167(1)$ & $0.003(2)$ & $0.005(2)$ & $3.050(8)$ \\
\hline
\end{tabular}




\section{Figure Captions:}

Figure 1: Schematic layout of the new high pressure beamline with a superbend dipole magnet source. The beamline acceptance is $1.0 \times 0.22 \mathrm{mrad}(\mathrm{h} \mathrm{x} \mathrm{v})$. The toroidal M2 mirror demagnifies in the horizontal in a 2:1 ratio.

Figure 2: Schematic layout of the monochromator elements within the Kohzu monochromator.

Figure 3: Calculated reflectivity plots platinum, rhodium and two bilayers consisting of either 4 or $8 \mathrm{~nm}$ rhodium over $25 \mathrm{~nm}$ platinum. Zero angstrom roughness is assumed. The bilayer extends the energy range cut off beyond that for a simple rhodium mirror whilst reducing the effects of the platinum $\mathrm{L}$ absorption edges.

Figure 4: Schematic layout of the on axis sample viewing system and the scatter clean up pinholes.

Figure 5: Schematic drawing of the sample stage on end-station 1. 1: x-translation stage to center rotation axis on beam. 2: rotation stage; 3: y-stage for vertical alignment, 4: x-stage to place DAC-center on rotation axis and beam, z-stage to place DAC-center on rotation axis. The rotation axis is the reference position, which defines the distance to the detector. Once aligned onto the beam, its position remains fixed.

Figure 6. Schematic drawing of the sample centering procedure: The aim is to place the DAC exactly onto the rotation axis. To do this we need to find the off-set shift $\mathrm{z}$. By measuring the DAC position at $0^{\circ}$ and $\omega^{\circ}$ rotation relative to the X-ray beam (through scans of the sample hole across the beam), distance $\mathrm{dx}$ is determined. The off-set $\mathrm{z}$ is then calculated as $\mathrm{z}=$ $\mathrm{dx} / \tan \omega$. Moving the DAC by this amount along the $\mathrm{z}$-stage will place it on the rotation axis. The same procedure can also be used to place a calibrant onto the rotation axis, which is used to determine the distance between rotation axis and detector plane.

Figure 7: Calculated and measured total flux of the beamlines at the sample position . Flux rates are for $400 \mathrm{~mA}$ in the ALS and full acceptance of the beamline resulting in a convergence onto the sample of $2.0 \mathrm{mrad}(\mathrm{h}) \mathrm{x} 0.15 \mathrm{mrad}(\mathrm{v})$. 
Figure 8: Comparison between pressures measured using the ruby fluorescence method with pressure determined with the help of $\mathrm{NaCl}$ as internal standard. 
Figure 1:
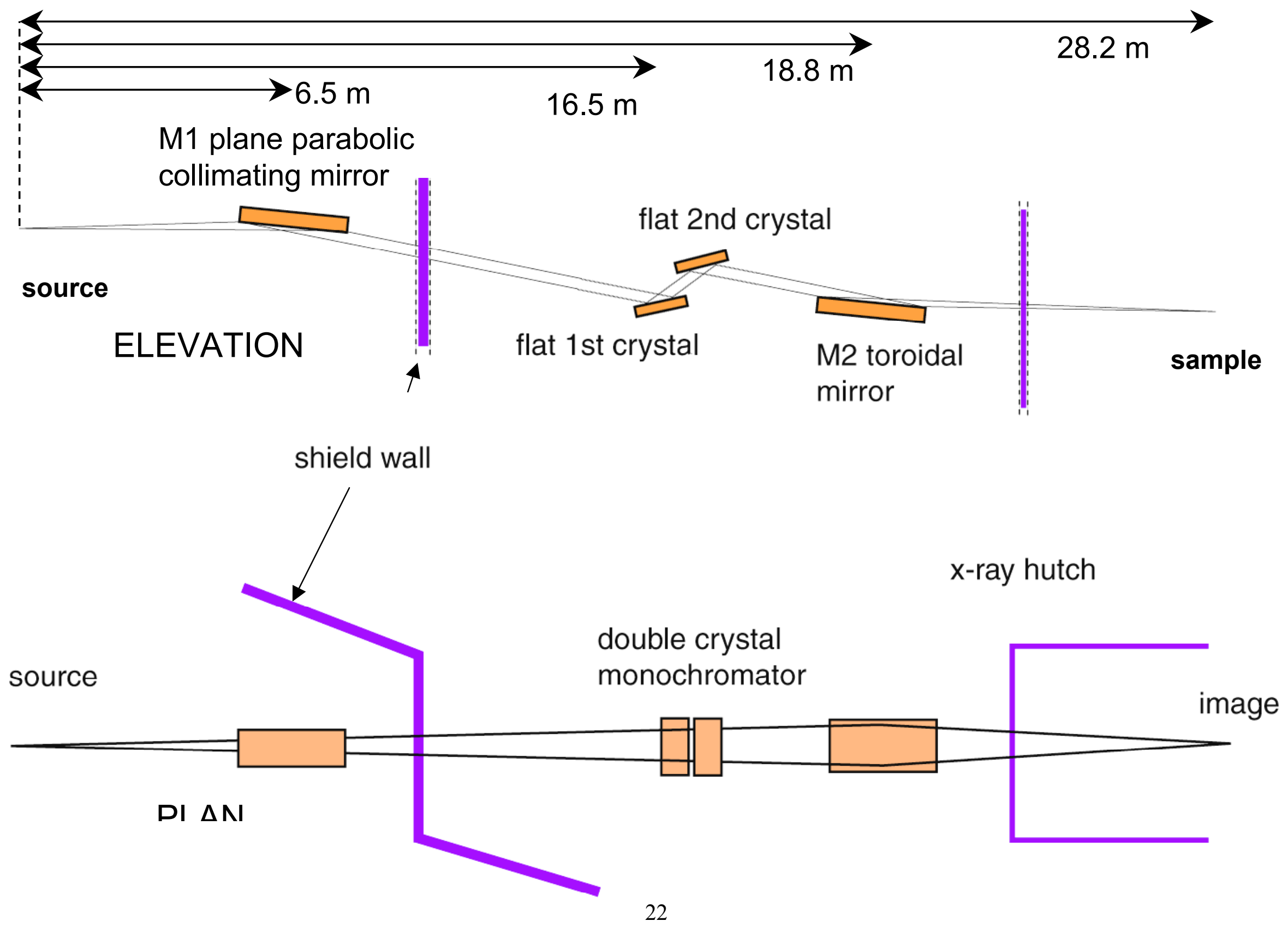
Figure 2:
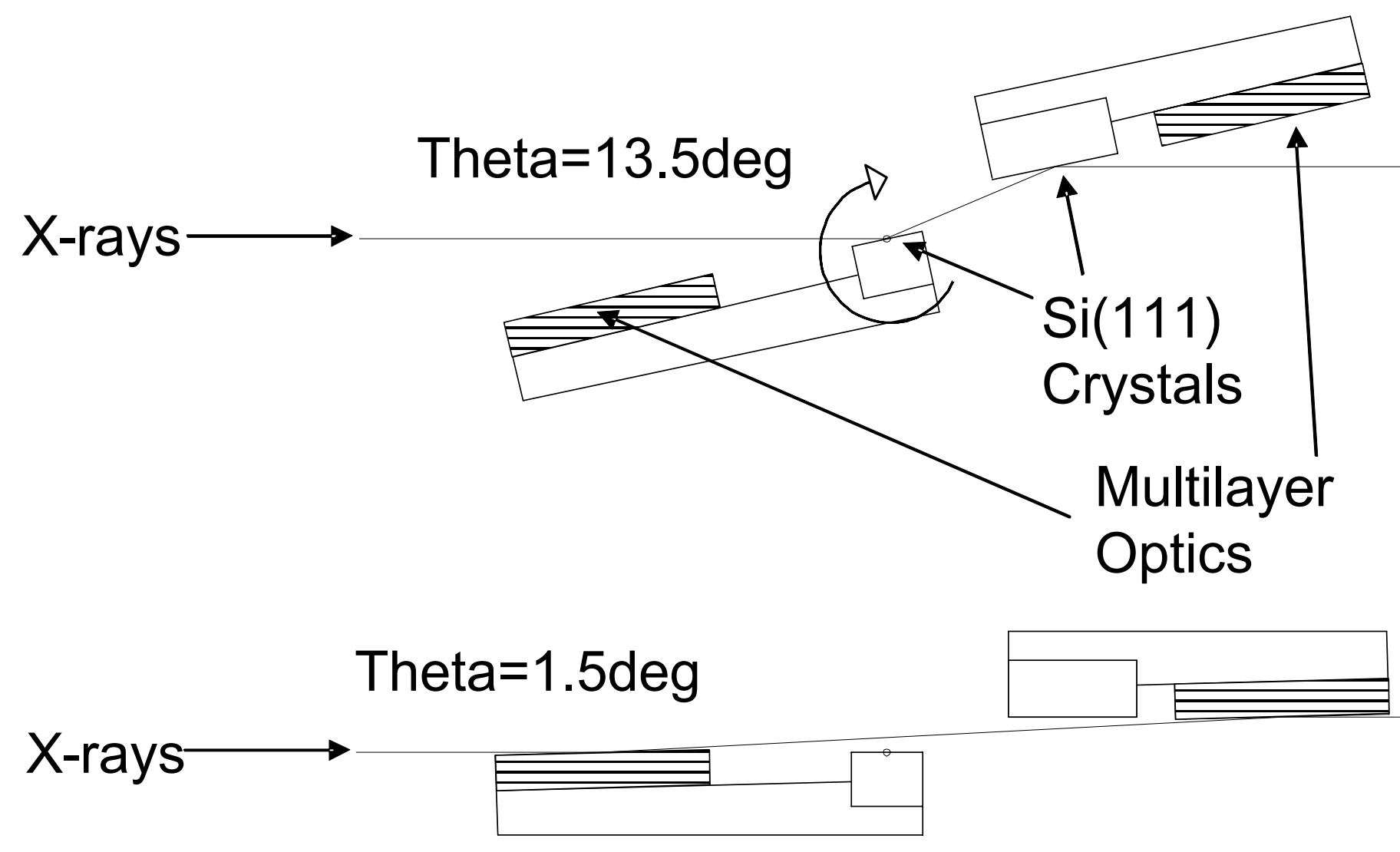
Figure 3:

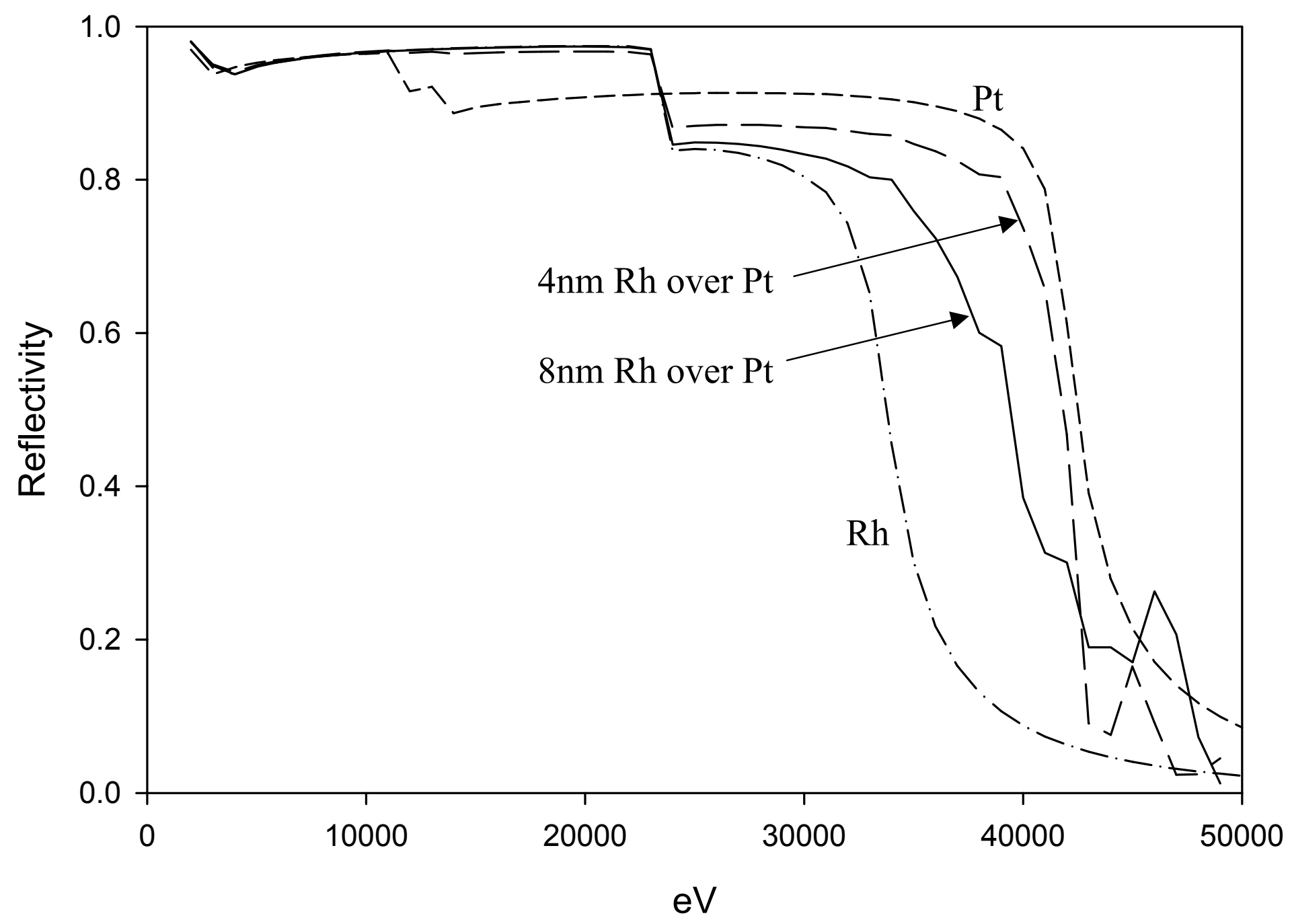


Figure 4:

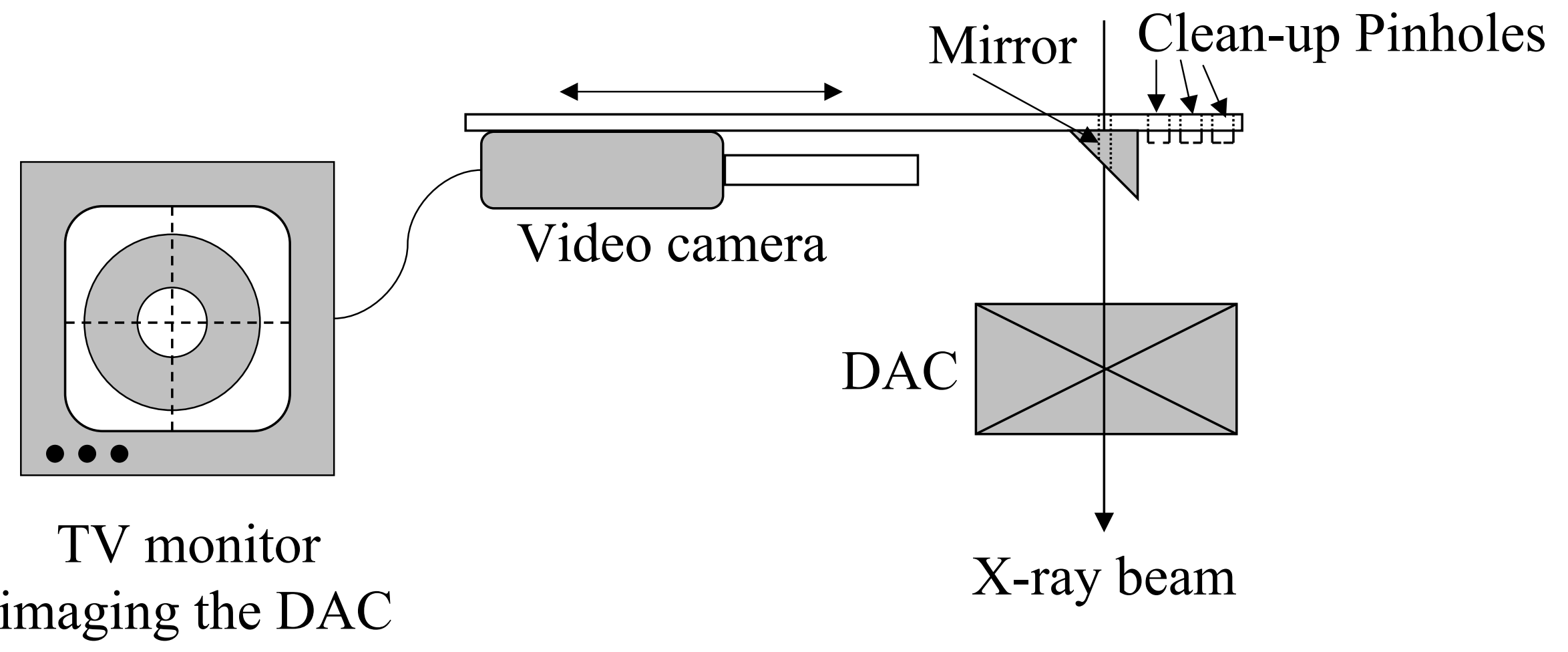


Figure 5:

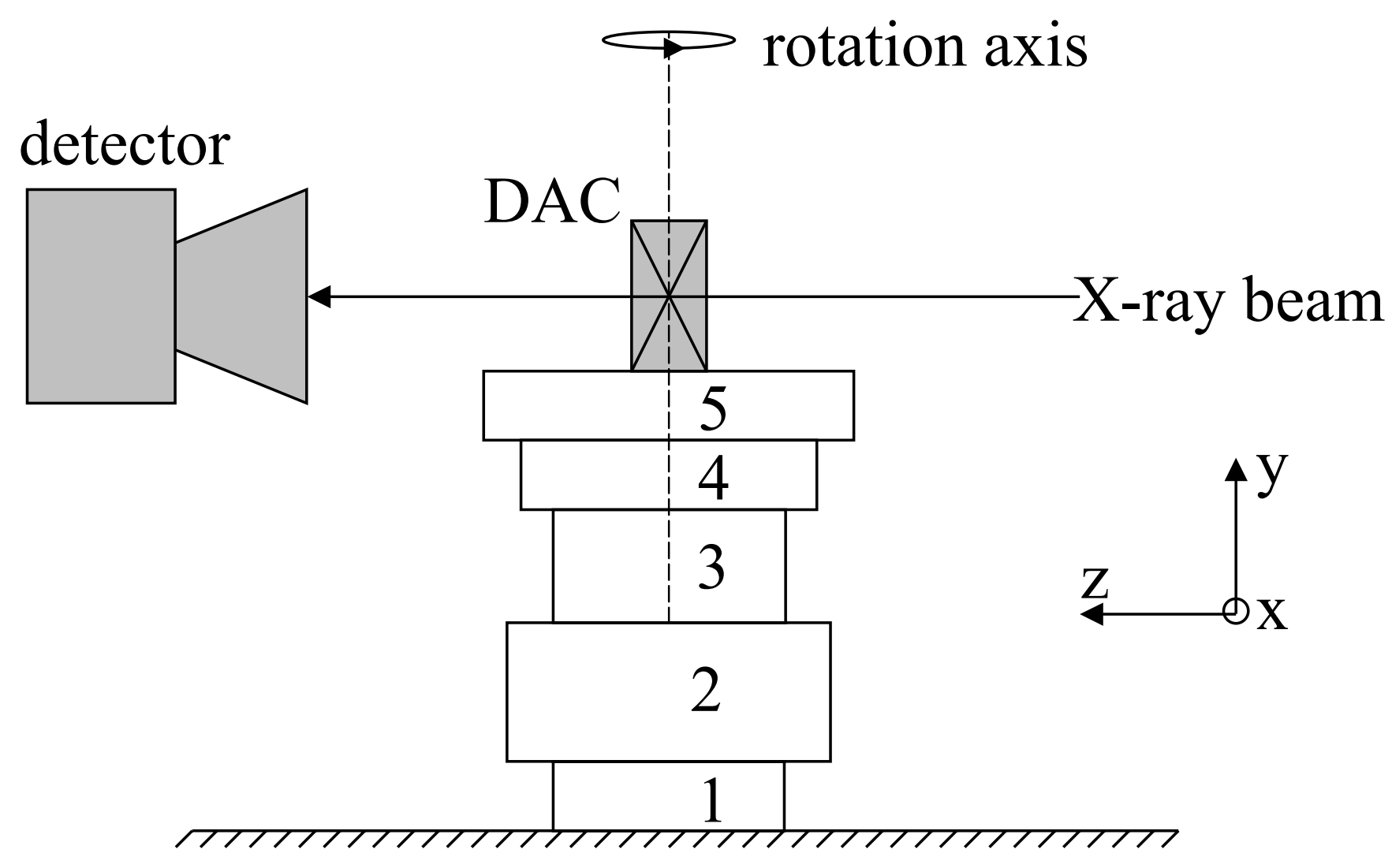


Figure 6:

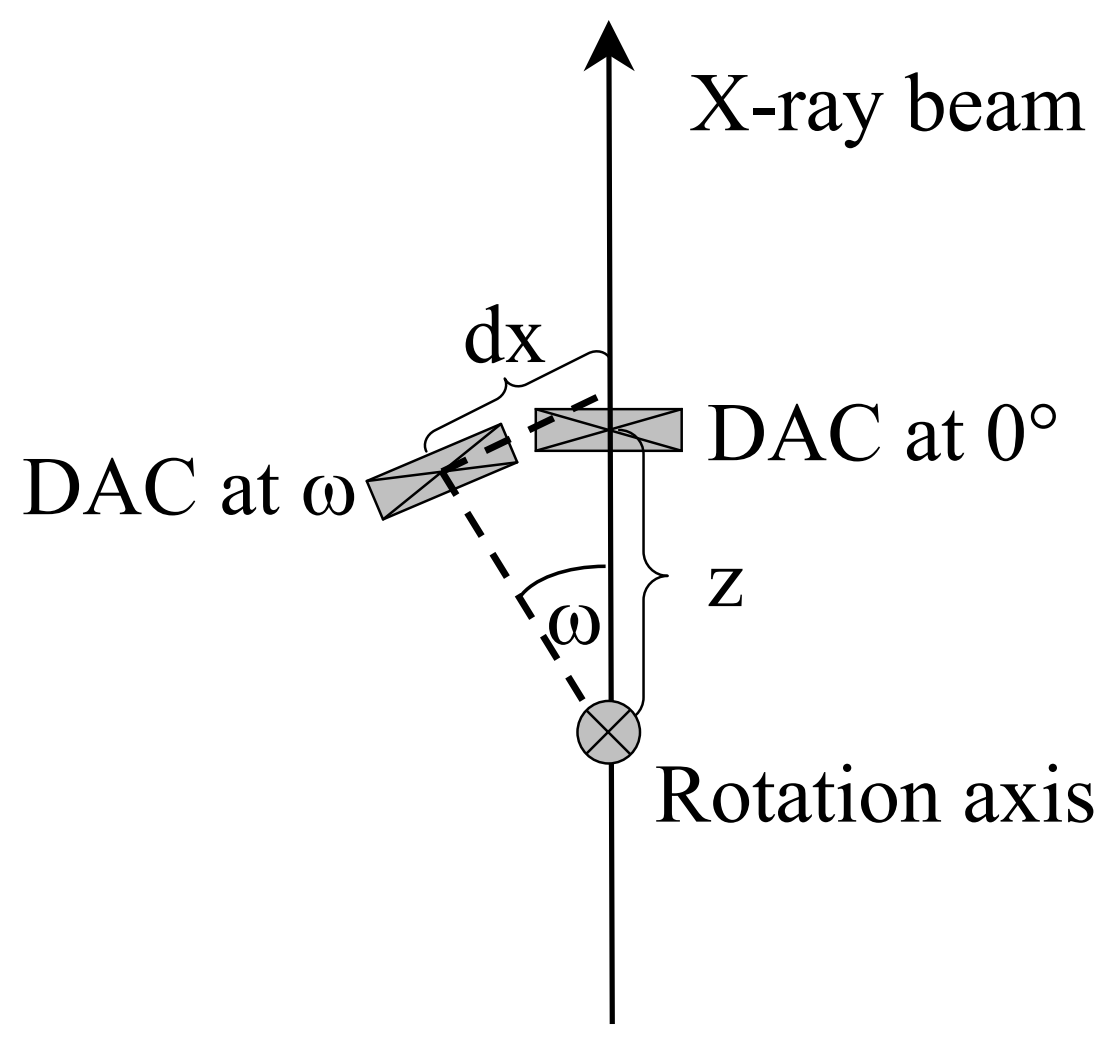


Figure 7:

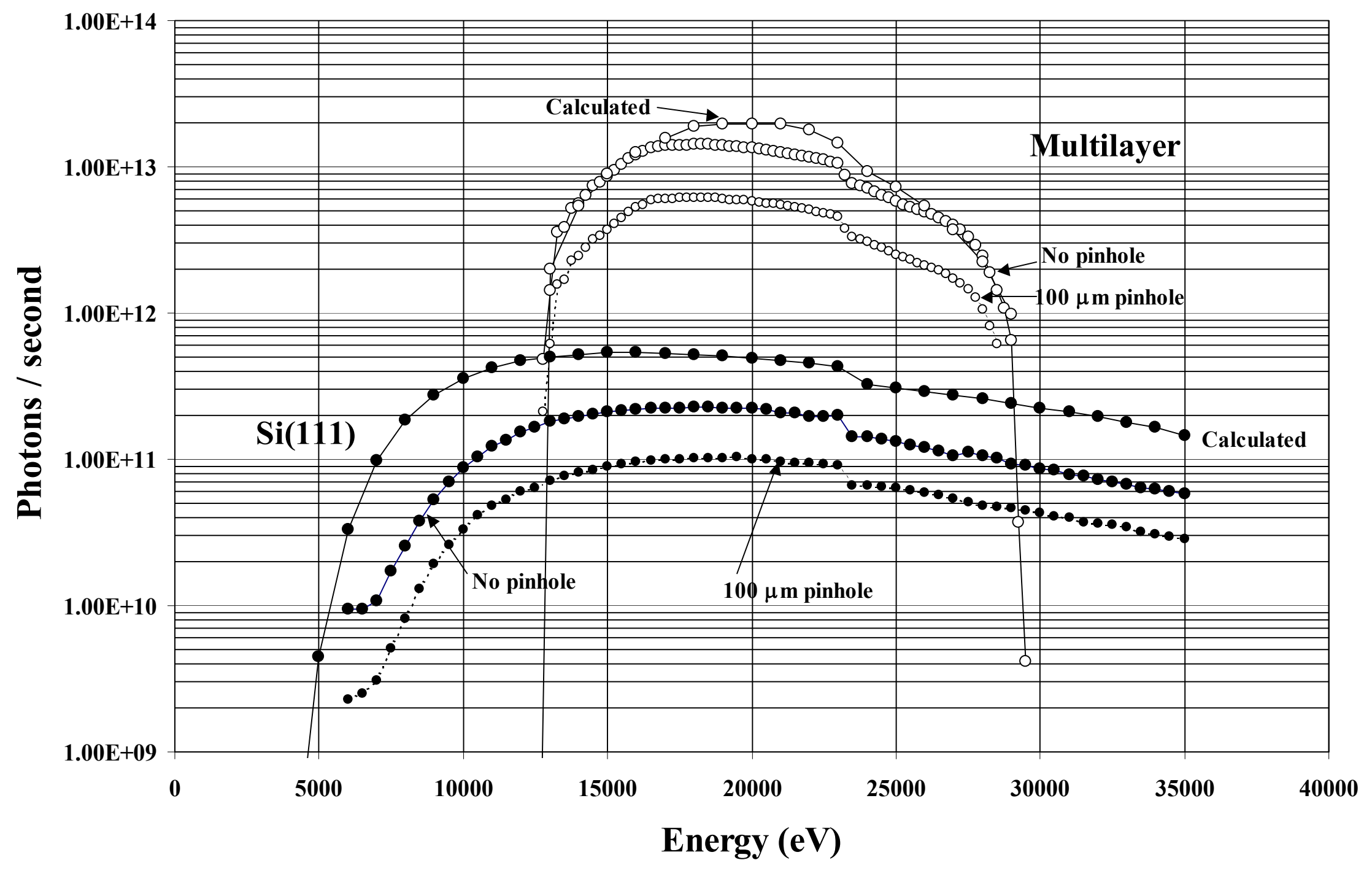


Figure 8:

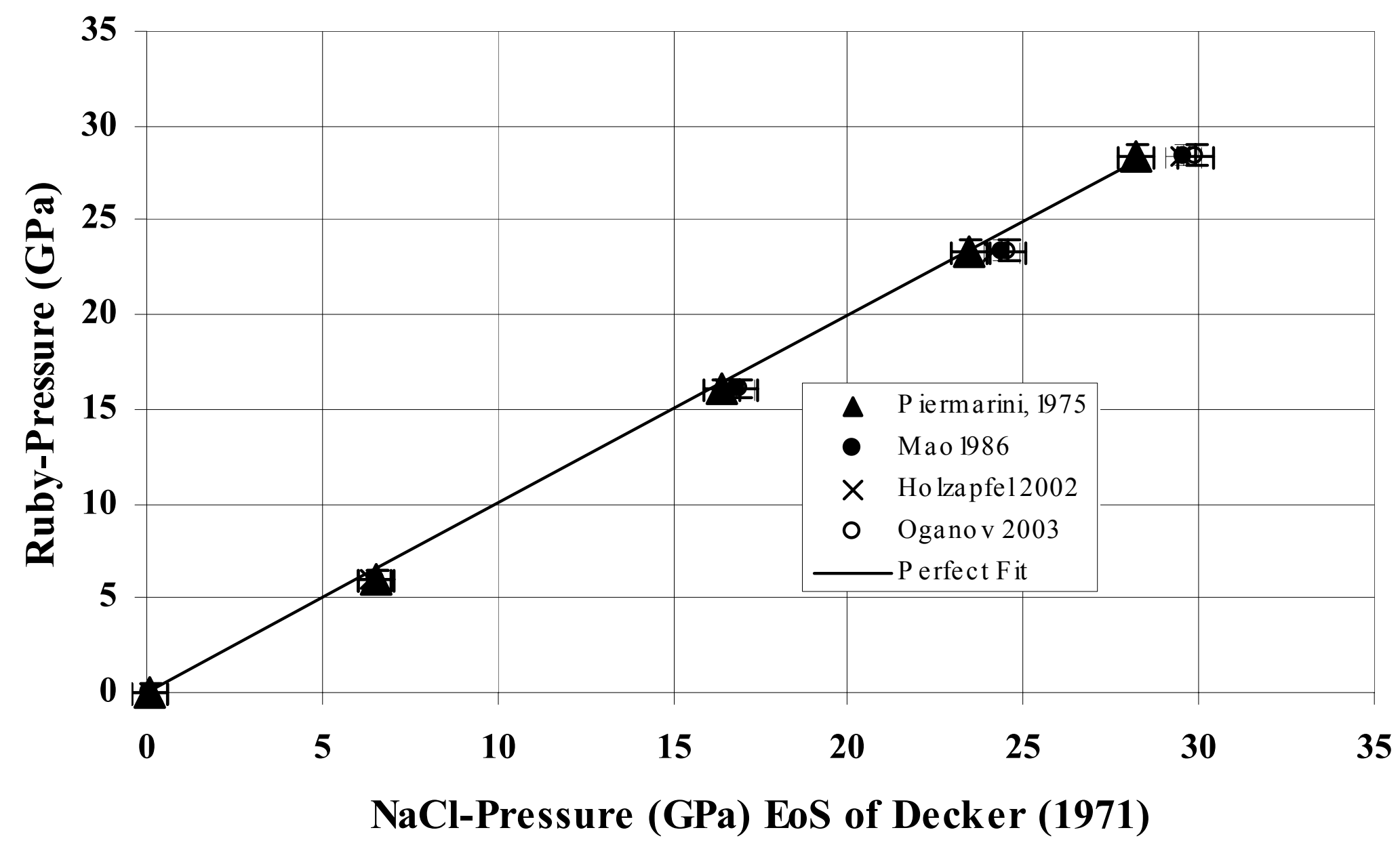

